\title{
How much troponin leak is too much before CABG?
}

\author{
Clancy Mullan ${ }^{1}$ and Arnar Geirsson ${ }^{2}$ \\ ${ }^{1}$ Yale School of Medicine \\ ${ }^{2}$ Yale University School of Medicine
}

March 25, 2021

How much troponin leak is too much before CABG?

Clancy W. Mullan, MD and Arnar Geirsson, MD

Division of Cardiac Surgery

Department of Surgery

Yale School of Medicine

330 Cedar Street, BB204

New Haven, CT 06511

Acute myocardial infarction (MI) is a well-recognized risk factor for worse outcomes after coronary artery bypass grafting (CABG), and the proportion of patients undergoing CABG after MI appears to be increasing over time(1). With nearly a third of patients undergoing CABG having presented with MI, the question has been asked repeatedly of whether the degree of troponin leak correlates to post-operative outcomes, with conflicting results through the years $(2,3)$. In this edition of the Journal of Cardiac Surgery, Dr. Hess and colleagues present a compelling argument against the use of the degree of troponinemia in predicting major outcomes of surgical revascularization after non-ST elevation MI (NSTEMI). Principally, the authors demonstrate that neither troponin I leak above median nor increasing troponinemia independently predict mortality or major adverse cardiac or cerebrovascular events (MACCE). Secondarily, they find that multivariable adjustment obviates the association of peak troponin level with prolonged ventilation, prolonged intensive care unit stay, and prolonged hospital stay.

With these data, the reader must ask: does there exist a residual argument for delaying surgical revascularization? A key observation in the present study is that nearly the entire cohort underwent urgent, rather than emergent or elective, revascularization. In the context of this relatively large cohort, this suggests a degree of stability to the "average" NSTEMI patient. Furthermore, given that the time from peak troponin to revascularization did not differ between the low- and high-risk cohorts and that time from peak troponin to surgery was not associated with post-operative mortality or MACCE, the data reported argue that patients can afford to wait for optimization prior to proceeding to surgery, certainly a controversial topic with a notable lack of society-level guidance that the authors appropriately address in their discussion. While the 2014 American Heart Association/American College of Cardiology guidelines for management of patients with NSTEMI provides some guidance on timing of surgical revascularization in relation to P2Y12 antiplatelet agent administration, no recommendations are provided on when to proceed with CABG otherwise. The authors findings seemingly fit directly on the middle of the debate's metaphorical fence; however, interpretation of this is challenging given a lack of information such as time from presentation to peak troponin level, from presentation to coronary catheterization, or from catheterization to surgery. 
What should, then, dictate when to take a patient with NSTEMI to the operating room? There is no straightforward answer to this question, but, generally, these patients should proceed to surgery soon enough to prevent progression from a non-transmural to a transmural injury but late enough to avoids the bleeding risk of potent P2Y12 inhibitor loads $(4,5)$. While emergent revascularization is likely not warranted in the absence of arrythmias or evidence of continued ischemia, urgent revascularization within 24 hours should be encouraged barring prohibitive bleeding risk from an antiplatelet agent load. Interestingly, the authors did not find intra-aortic balloon pump (IABP) placement to be associated with post-operative mortality hazard, suggesting that IABPs were not especially targeted to unstable patients in their population. However, pre-operative inotrope requirement was associated with increased post-operative mortality, supporting an argument that NSTEMI patients, like their STEMI counterparts, with cardiogenic shock represent a particularly vulnerable population that should be revascularized with greater urgency. Where coronary anatomy demands surgical revascularization and cardiac surgical resources are not available, medical optimization followed by prompt referral to a surgical center is key.

The tenacity with which the authors undertook the principal analyses of the manuscript should be commended. Hess et al. present a thorough and convincing argument that a patient's risk from NSTEMI is likely fixed and dictated by the overall clinical picture rather than dependent on the degree of troponinemia and that the peak troponin level should not dictate clinical decisions.

\section{References}

1. Alkhouli M, Alqahtani F, Kalra A et al. Trends in characteristics and outcomes of hospital inpatients undergoing coronary revascularization in the united states, 2003-2016. JAMA Network Open 2020;3(2):e1921326-e1921326.

2. Beller JP, Hawkins RB, Mehaffey JH et al. Does preoperative troponin level impact outcomes after coronary artery bypass grafting? The Annals of thoracic surgery 2018;106(1):46-51.

3. Thielmann M, Massoudy P, Neuhäuser M et al. Prognostic value of preoperative cardiac troponin i in patients undergoing emergency coronary artery bypass surgery with non-st-elevation or st-elevation acute coronary syndromes. Circulation 2006;114(1 Suppl):I448-453.

4. Amsterdam EA, Wenger NK, Brindis RG et al. 2014 aha/acc guideline for the management of patients with non-st-elevation acute coronary syndromes: A report of the american college of cardiology/american heart association task force on practice guidelines. Circulation 2014;130(25):e344-426.

5. Hillis LD, Smith PK, Anderson JL et al. 2011 accf/aha guideline for coronary artery bypass graft surgery. Journal of the American College of Cardiology 2011;58(24):e123-e210. 